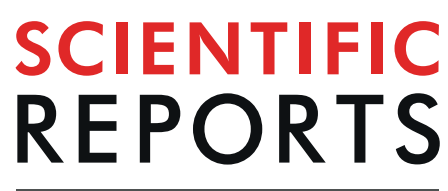

natureresearch

\title{
Grey mould control by oxalate degradation using non-antifungal Pseudomonas abietaniphila strain ODB36
}

\author{
Yeyeong Lee ${ }^{1}$, Okhee Choi ${ }^{2}$, Byeongsam Kang ${ }^{3}$, Juyoung Bae ${ }^{3}$, Seunghoe Kim ${ }^{1}$ \& \\ Jinwoo Kim $\mathbb{1}^{1,2,3^{*}}$
}

Grey mould is an important necrotrophic fungal pathogen that causes huge economic losses in agriculture. Many types of bacteria are used for biological control of grey mould via competition for space or nutrients and/or the production of antifungal metabolites. Oxalate is a key component of virulent necrotic fungal pathogens. In this study, we isolated non-antifungal oxalate-degrading bacteria (ODB) from the surfaces of oxalate-rich spinach and strawberries to investigate their ability to control necrotic fungal pathogens such as grey mould. A total of 36 bacteria grown on oxalate minimal (OM) agar plates were tested for oxalate-degrading activity. Five isolates exhibiting the highest oxalate degradation activity were subjected to molecular identification using $16 \mathrm{~S}$ rRNA gene sequencing. Two isolates exhibiting non-antifungal activity were subjected to disease suppression assays using Arabidopsis-Botrytis systems. The isolate Pseudomonas abietaniphila ODB36, which exhibited significant plant protective ability, was finally selected for further investigation. Based on wholegenome information, the $\underline{p s e u d o m o n a d} \underline{\underline{o x a l a t e}} \underline{\mathrm{d} e g r a d i n g}$ (podA) gene, which encodes formyl-CoA transferase, was analysed. The podA- mutant did not inhibit Botrytis infection and oxalate toxicity; the defects were recovered by podA complementation. Purified PodA-His converted oxalate to formate and eliminated oxalate toxicity. These results indicate that P. abietaniphila ODB36 and PodA enzyme are associated with various aspects of grey mould disease inhibitory effects.

Botrytis cinerea, which causes grey mould, is an important necrotrophic pathogen that causes huge economic losses in agriculture ${ }^{1}$. Grey mould is known to attack over 200 crop hosts including vegetables, fruits, flowers, and post-harvest crops ${ }^{1}$. Grey mould can cause soft rotting of all aerial plant parts and produce prolific gray conidiophores and conidia typical of the disease ${ }^{1}$. B. cinerea causes seed contamination in flax, sunflower, and lettuce ${ }^{2}$. In Australia, seed contamination by $B$. cinerea has caused total crop failure in chickpea ${ }^{3}$.

Although agricultural systems typically rely on chemical pesticides to inhibit grey mould, fungicides are avoided in sustainable agriculture due to their effects on the well-being of humans and the surrounding environment ${ }^{4}$. Fungicide overuse has led to the emergence of resistant fungal strains ${ }^{5}$. During the past few decades, these issues have led many researchers to develop biological control methods using antagonistic bacteria. The modes of antagonistic bacterial activity include competition, antibiosis, lytic enzyme production, interference with pathogen activity and growth, volatility, and host resistance induction. However, most biological controls evaluated under laboratory conditions have tended to fail in the field ${ }^{6}$. Sulfur dioxide fumigation is usually used to control postharvest grey mould; however, because it decreases berry quality by absorption in detached berries, safe, effective, and economical alternatives are needed ${ }^{7}$.

Many necrotrophic pathogens including grey mould are known to infect various host plants via the production of oxalate, which is toxic to cellular organisms including mammals and plants ${ }^{8,9}$. Oxalate acts as a strong chelator of cations; it greatly oxidizes organic compounds in necrotrophic pathogens ${ }^{10}$, helps to reduce $\mathrm{pH}$, thereby facilitating plant infection by pectolytic enzymes, and impedes defense signaling pathways in the host ${ }^{11}$. Therefore, degrading oxalate at the onset of host pathogen interaction should block the infection process. Oxalate-degrading

${ }^{1}$ Department of Plant Medicine, Gyeongsang National University, Jinju, 52828, Republic of Korea. ${ }^{2}$ Institute of Agriculture \& Life Science, Gyeongsang National University, Jinju, 52828, Republic of Korea. ${ }^{3}$ Division of Applied Life Science, Gyeongsang National University, Jinju, 52828, Republic of Korea. *email: jinwoo@gnu.ac.kr 


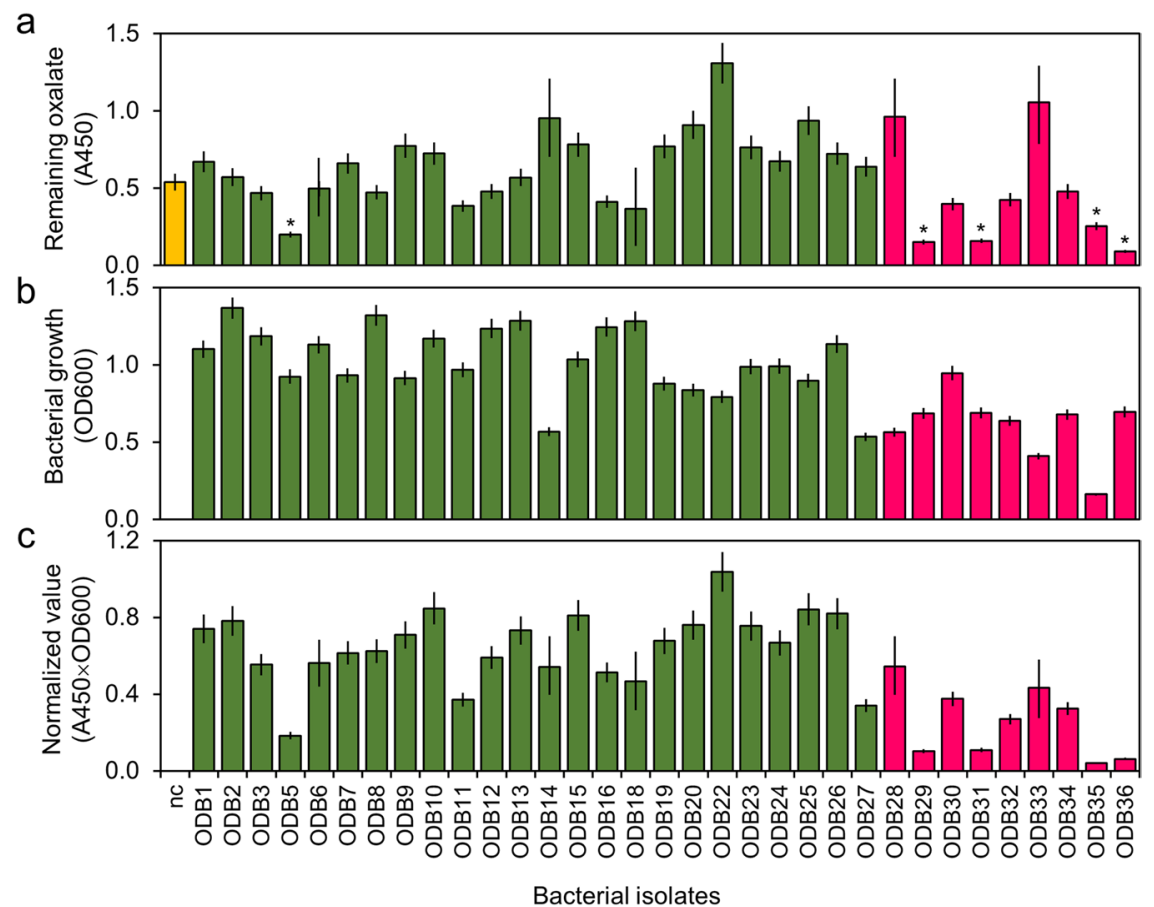

Figure 1. Oxalate degradation by bacterial isolates. (a) Absorbance measurement at $\mathrm{A}_{450}$. Oxalate in the supernatants was determined using an oxalate colorimetric assay kit. Bacterial isolates were grown in oxalate minimal (OM) medium supplemented with $1 / 10 \mathrm{TSB}$ at $28^{\circ} \mathrm{C}$ for 3 days. Cell-free supernatants were subjected to the sample preparation protocols described in Methods. The values report the amount of oxalate remaining in the OM medium. A total of 36 bacteria growing on OM agar plates were isolated from the surfaces of spinach (27 isolates; green) and strawberries (9 isolates; red). (b) Optical density $\left(\mathrm{OD}_{600}\right)$ of the bacterial growth. (c) Normalised values calculated by multiplying the $\mathrm{A}_{450}$ value of the oxalate amount by the $\mathrm{OD}_{600}$ of the culture. The lower the value, the higher the degradation activity. Bacterial isolates ODB5, 29, 31, 35, and 36 exhibited significant oxalate degradation. Values are averages of triplicate assays and error bars represent the range. nc denotes the non-inoculated medium control. Asterisks denote significant differences from the control $\left({ }^{*} p<0.05\right.$; Student's $t$ test).

microorganisms are useful alternatives to chemicals or fungicides. Oxalate-quenching Pseudomonas fluorescens PfMDU2 was shown to inhibit the mycelial growth of Rhizoctonia solani, which causes rice sheath blight ${ }^{12}$. The oxalate-degrading bacterial strain Cupriavidus campinensis was reported to protect Arabidopsis thaliana and crop species against $B$. cinerea $^{13}$.

In human, oxalates are consumed when eating foods high in oxalates such as spinach, strawberries, coffee, tea, and chocolate, or are produced by intestinal microorganisms from metabolic precursors. In the human intestine, oxalate can combine with calcium, magnesium, potassium, or sodium to form less-soluble salts, which can lead to pathophysiological disorders, including hyperoxaluria, urolithiasis, and kidney failure ${ }^{14,15}$. To overcome these disorders, studies on the use of oxalate-degrading enzymes of intestinal microorganisms such as Oxalobacter formigenes and Lactobacillus acidophilus have been conducted ${ }^{15,16}$.

The objective of this study was to isolate, characterize, and evaluate oxalate-degrading bacteria (ODB) for the control of grey mould. We performed protein overexpression of the pseudomonad oxalate $\underline{\text { degrading enzyme }}$ (PodA) from non-antifungal Pseudomonas abietaniphila. Our aim was to provide a new approach for the control of grey mould via the application of oxalate-degrading genetic resources.

\section{Results}

ODB isolation and oxalate-degrading activity. A total of 36 bacteria grown on oxalate minimal (OM) agar plates $\left(2 \mathrm{~g} \mathrm{Na}_{2} \mathrm{C}_{2} \mathrm{O}_{4}, 2.7 \mathrm{~g} \mathrm{~K}_{2} \mathrm{HPO}_{4}, 0.9 \mathrm{~g} \mathrm{NaH}_{2} \mathrm{PO}_{4}, 0.9 \mathrm{~g} \mathrm{NH}_{4} \mathrm{Cl}, 0.27 \mathrm{~g} \mathrm{MgSO}_{4} \cdot 7 \mathrm{H}_{2} \mathrm{O}, 0.009 \mathrm{~g} \mathrm{CaCl}_{2} \cdot 2 \mathrm{H}_{2} \mathrm{O}\right.$, and $0.0024 \mathrm{~g} \mathrm{FeSO}_{4} \cdot 7 \mathrm{H}_{2} \mathrm{O}$ in $1 \mathrm{~L}$ distilled water with $1.5 \%$ agar) were isolated from the surfaces of spinach $(27$ isolates) and strawberries ( 9 isolates). The oxalate-degrading activity of these 36 bacteria was tested in OM broth supplemented with $1 / 10$ Bacto tryptic soy broth (TSB). Oxalate in the culture supernatants was determined using an oxalate colorimetric assay kit (Bio Vision Inc., San Francisco, CA, USA). Therefore, the lower the absorbance value at $450 \mathrm{~nm}\left(\mathrm{~A}_{450}\right)$, the higher the degradation activity. When the non-inoculated media control (nc) supplemented with $15 \mathrm{mM}$ sodium oxalate showed a value of 0.55 , five isolates (ODB5, ODB29, ODB31, ODB35, and ODB36) exhibited significantly reduced values $<0.5$ (Fig. 1a). Isolates with values higher than the control (nc) were suspected to have the ability to synthesize oxalate by themselves, and thus exhibited values $>0.55$ (Fig. 1a). Each isolate showed a different growth pattern; in particular, the growth of ODB35 was relatively slow compared to other isolates (Fig. 1b). Because the growth of each isolate directly affects oxalate degradation, it is necessary to normalize values. Five isolates (ODB5, ODB29, ODB31, ODB35, and ODB36) had normalized values $<0.2$, 
a Bacterial treatment $(\mathrm{OD} 600)$

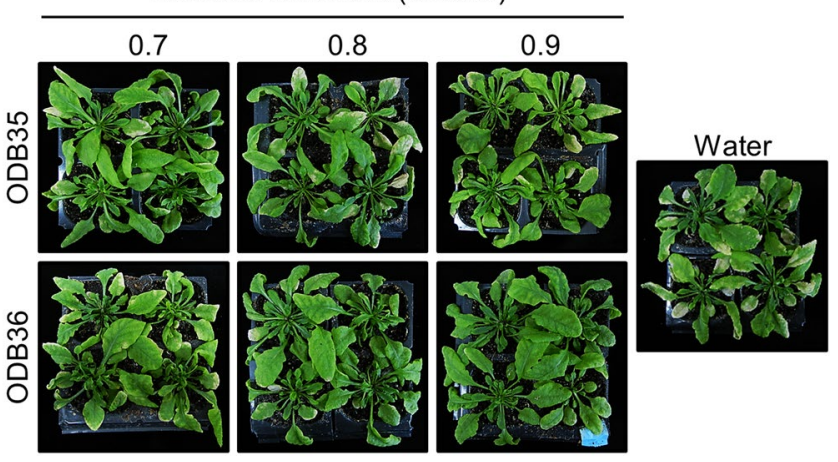

b

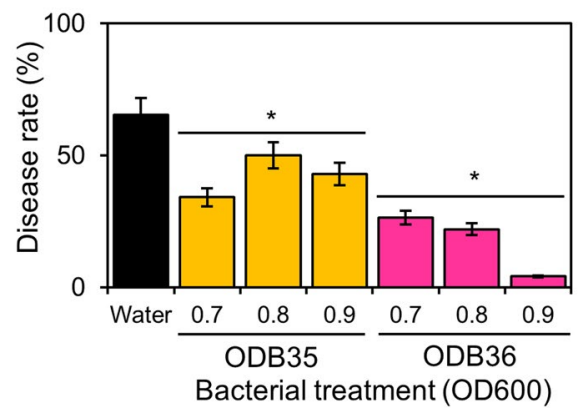

Figure 2. Grey mould disease suppression by ODB35 and ODB36. (a) Image captured 16 days after inoculation; (b) disease rate (\%). Three-week-old Arabidopsis plants were sprayed with the ODB suspension. After incubation for 2 days, a $B$. cinerea spore suspension $\left(5 \times 10^{5}\right.$ spores $\left./ \mathrm{mL}\right)$ was prepared using sterilised water. The spore suspension was sprayed until run-off occurred. Inoculated plants were placed in an opaque plastic box lined with saturated paper towels, the lids were removed after 2 days, and disease development was observed for 16 days and measured. ODB36 exhibited significant disease suppression depending on treatment concentration. Values are averages of triplicate assays; error bars represent the range. Asterisks denote significant differences from the water control $\left({ }^{*} p<0.05\right.$; Student's $t$ test).

indicating that they had considerable oxalate-degrading activity (Fig. 1c). Three isolates (ODB4, ODB17, and ODB21) that did not grow in liquid culture were excluded.

Bacterial identification and antifungal activity tests. To confirm the identities of the ODB, we amplified and sequenced the 16S rRNA genes of ODB5, ODB29, ODB31, ODB35, and ODB36, which exhibited significant oxalate-degrading activity. BLAST analysis of the $16 \mathrm{~S}$ rRNA gene sequence of ODB5 showed 99\% identity with P. fluorescens; those of ODB29, ODB31, and ODB36 showed 99\% identity with P. abietaniphila; and that of ODB35 showed 99\% identity with Methylobacterium zatmanii.

Isolates ODB5, ODB35, and ODB36 were selected for further antifungal activity testing. ODB5 exhibited antifungal activity against B. cinerea, Alternaria alternata, and Saccharomyces cerevisiae, whereas ODB35 and ODB36 showed no antifungal activity (see Supplementary Fig. S1).

Disease suppression assay. To investigate disease suppression of ODB35 and ODB36, which exhibited no antifungal activity, we used the standard A. thaliana-B. cinerea (plant-pathogen) system. After 16 days of inoculation, water-sprayed plants exhibited a 65\% disease rate against grey mould, whereas ODB36-sprayed plants exhibited significant disease suppression depending on the treatment concentration (Fig. 2). However, ODB35 exhibited no significant disease suppression compared with ODB36 (Fig. 2).

Phylogenetic analysis and sequence alignment. Using whole-genome information from GenBank (accession no. SDG16549), we analyzed a gene encoding an oxalate-degrading enzyme in P. abietaniphila ATCC $700689^{17}$. Translated amino acid sequences of PodA ( $42 \mathrm{kDa}$ ) showed $98 \%$ similarity with P. abietaniphila ATCC 700689 and $82 \%$ with P. fluorescens BBc6R8. Figure 3 shows the phylogenetic relationships of several putative formyl-CoA transferases from organisms whose protein sequences were available. As expected, the formyl-CoA transferases from P. abietaniphila and P. fluorescens clustered together. Sequence analysis showed that PodA, a formyl-CoA transferase, belongs to pfam02515, the CaiB-BaiF family of enzymes with diverse functions including fatty acid racemases, carnitine dehydratase (CaiB), bile acid inducible operon protein $\mathrm{F}(\mathrm{BaiF})$, benzylsuccinate CoA-transferase (BbsF), and anaerobic toluene catabolic protein in the presence of toluene. Identical residues, shown in red, are located in half of the $\mathrm{N}$-termini of the proteins and partially in the C-termini (Fig. 4). 


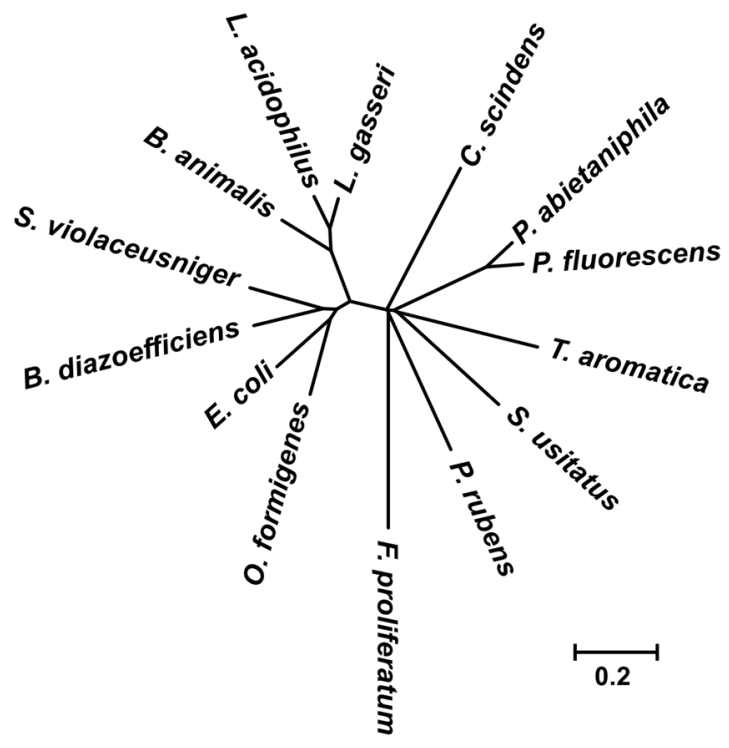

Figure 3. Unrooted phylogenetic tree of formyl-CoA transferase sequences. Proteins were aligned using CLUSTALX. Alignments were used for tree reconstruction. The organisms used were Bifidobacterium animalis (GenBank accession number WP004219152), Bradyrhizobium diazoefficiens USDA110 (NP769796), Clostridium scindens (AAC45415), Escherichia coli CFT073 (WP000106774), Fusarium proliferatum (AMB48876), Lactobacillus acidophilus NCK1728 (AAV42286), Lactobacillus gasseri ATCC 3323 (WP144231654), Oxalobacter formigenes Ox-B (AAC45298), Penicillium rubens Wisconsin 54-1255 (CAP92691), Pseudomonas fluorescens BBc6R8 (WP003209938), Solibacter usitatus Ellin 6076 (ABJ84069), Streptomyces violaceusniger Tu 4113 (AEM82356), and Thauera aromatica (AAF89841).

PodA is required for the inhibition of Botrytis infection. To investigate the effect of the wild-type ODB36, podA ${ }^{-}$(podA-lacZY) mutant, and podA complementation strains on Botrytis infection, we performed disease suppression assays. Wild-type strain exhibited significant disease suppression. The pod $A^{-}$mutant did not inhibit Botrytis infection; the defect was recovered by podA complementation (Fig. 5). These results indicated that podA is critical for the inhibition of Botrytis infection in P. abietaniphila.

PodA is required for the inhibition of oxalate toxicity. In tobacco leaf disc assays, oxalate toxicity, such as bleaching of leaf discs, was observed for the podA $A^{-}$(podA-lacZY) mutant but was weak for the wild-type and podA complementation strains (Fig. 6a). This result was confirmed by the quantification of chlorophyll. Tobacco leaf discs treated with the podA- mutant showed bleaching or a low chlorophyll level. These defects were restored to near wild-type levels by podA complementation (Fig. 6b). Also, oxalate degradation was induced by treatment of the wild-type and podA complementation strains (Fig. 6c).

Overexpression, purification, and oxalate-degrading and formate conversion activities of PodA protein. We overexpressed and purified P. abietaniphila ODB36 PodA from Escherichia coli BL21(DE3) carrying pLY205 (pET21b::podA). The podA gene encodes a protein of 396 amino acid residues with a calculated molecular weight of $42 \mathrm{kDa}$. To confirm that the podA gene encodes an oxalate-degrading enzyme, it was expressed under control of the T7 promoter in E. coli. Upon induction of E. coli BL21(DE3) harbouring pLY205 with IPTG, a His-tagged protein with a molecular mass of around $42 \mathrm{kDa}$ was produced; approximately half of the PodA-His protein was expressed in soluble bodies at pH 8.0-9.0 (Fig. 7a). PodA-His was purified to near homogeneity using Ni-NTA affinity chromatography (Fig. 7b). The oxalate colorimetric assay kit revealed that PodA-His $(0.8 \mathrm{mM})$ exhibited $75 \%$ oxalate-degrading activity (Fig. $7 \mathrm{c})$. Using a formate colorimetric assay kit, we measured the formate conversion activity of PodA-His; the amount of degradation confirmed that oxalate was consistently converted to formate (Fig. 7c).

Inhibition of oxalate toxicity by PodA-His protein. To investigate the inhibitory effect of PodAHis protein on oxalate toxicity, we used a standard A. thaliana-oxalate (plant-toxin) system. Oxalate-sprayed plants exhibited an $81 \%$ oxalate toxicity rate, while PodA-His-sprayed plants showed significant and concentration-dependent inhibition of oxalate toxicity (Fig. 8).

\section{Discussion}

Grey mould causes damage during the cultivation, storage, and transportation of fresh vegetables and soft-pulling fruits $^{1-3}$. Efforts have been made to control grey mould using antifungal bacteria, but this is complicated by the stability of antimicrobial substances in fresh vegetables and the emergence of resistance ${ }^{4,5}$. For this reason, we investigated the possibility of suppressing grey mould using non-antifungal ODB, similar to the use of a non-antagonistic bacterium to control Salmonella enterica, which causes food poisoning ${ }^{18}$. 


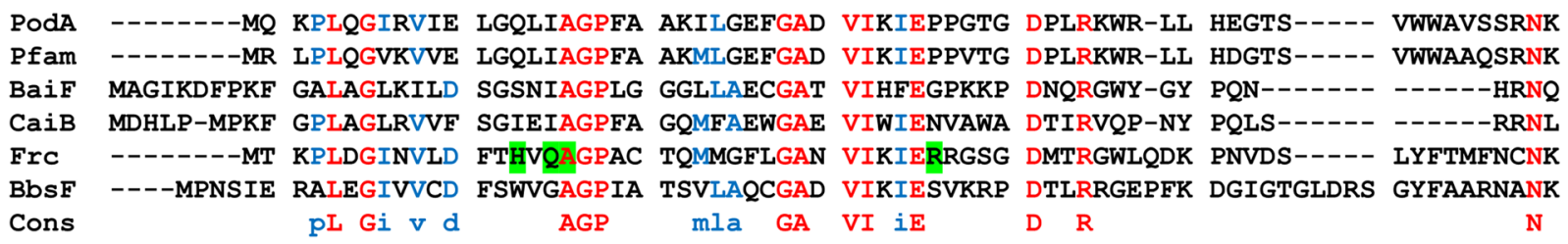

POdA RSVTLDLREP EGQDIVRKLI AEADVLVENF RPGTLEGWGL GWQELHALNP KLVMLRVSGY GQTG--PYRD RPGFGVVGEA Pfam RSLTLDLRQV EAQQVVRQLL ADADVLIENF RPGTLESWGL GWDTLHALNP KLVMLRVSGY GQTG--PYRD RPGFGVIGEA BaiF LSMVADIKSE EGRKIFLDII KWADIWVESS KGGQYDRLGL SDEVIWEVNP KIAIVHVSGY GQTGDPSYVT RASYDAVGQA CaiB HALSLNIFKD EGREAFLKLM ETTDIFIEAS KGPAFARRGI TDEVLWQHNP KLVIAHLSGF GQYGTEEYTN LPAYNTIAQA FrC RSIELDMKTP EGKELLEQMI KKADVMVENF GPGALDRMGF TWEYIQELNP RVILASVKGY AEGH--ANEH LKVYENVAQC BbSF RDIALDMSHP RAREVAVRLI EKSDIVINNF RVGQMEKWKL GWEDVQKINP RAIYVTMSMQ GIDG--PHSR YMGYGVNLNA

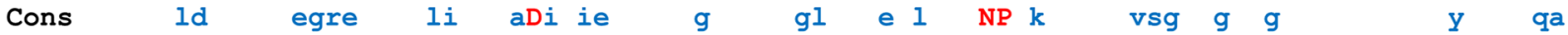

POdA MGGLRHLSGE PDRTPVRVGV SIGDSLSA-L HGVIGVLLAL RHRDQNGGEG QEVDVALYES VFNMMESLIP E--------Pfam MGGLRHLSGE PGRPPVRVGV SIGDSLSA-L HGVIGILLAL RHREQNGGDG QQIDVALYES VFNMMESLVP E--------BaiF FSGYMSLNG- -TTEALKINP YLSDFVCG-L TTCWAMLACY VSTILTG-KG ESVDVAQYEA LARIMDGRMI Q--------CaiB FSGYLIQNGD -VDQPMPAFP YTADYFSG-L TATTAALAAL HKARETG-KG ESIDIAMYEV MLRMGQYFMM D--------FrC SGGAAATTGF WDGPPTVSGA ALGDSNSG-M HLMIGILAAL EMRHKTG-RG QKVAVAMQDA VLNLVRIKLR DQQRLERTGI BbSF LCGLTARAGF PGQAPFGTGT NYTDHVMVPT HTLFGIMAAL LEREATG-RG QTVSLSQLES AICMTPSAPM A--------$\begin{array}{llllllll}G & G & p & D & g & \text { laal } & \text { tG } & \text { q v va e m }\end{array}$

PodA

Pfam

BaiF

Саiв

Frc

BbsF

Cons

------- -------F SVFGAVRQPA GS-SLPGIAP SNAYRCRDGR YALIAGNGDS IYQRLMALIE RPDL-AADPA ------- -------Y SVFNAVREPA GS-SLPGIAP TNAYRCRDGG FALIAGNGDS IYQRLMEVIG RRDL-GGDPD ----- -----Y ATDGVKMPRT GN-KDAQAAL FSFYTCKDGR TIFIGMTGAE VCKRGFPIIG LPVPGTGDPD -------- -------Y FNGGEMCPRM SKGKDPYYAG CGLYKCADG- YIVMELVGIT QIEECFKDIG LAHL-LGTPE LAEYPQAQPN FAFDRDGNPL SFDNITSVPR GGNAGGGGQP GWMLKCKGWE TDADSYVYFT IAANMWPQIC DMIDKPEWKD

$$
\begin{array}{lllllllll}
g & g & d & a & y & c & d & v & i
\end{array}
$$

POdA LAHN----D GRVKQVELID AAISAWAASL DLDEVLAKLT AERIPAGKIY DAADIASDPH YKARDMLLDS RLDDGTPVTL Pfam LAQN-----H GRVAQVERID AAISEWTARL TLDEVLSILT EARIPAGKIY NAADIAQDPH YRARDMLLDS QLDDGTAVTL BaiF FPEG-FTGWM IYTPVGQRME KAMEKYVSEH TMEEVEAEMQ AHQIPCQRVY ELEDCLNDPH WKARGTITEW DDPMMGHITG CaiB IPEGTQLIHR IECPYGPLVE EKLDAWLAAH TIAEVKERFA ELNIACAKVL TVPELESNPQ YVARESITQW QTMDGRTCKG FrC DPAYN--TFE GRVDKLMDIF SFIETKFADK DKFEVTEWAA QYGIPCGPVM SMKELAHDPS LQKVGTVVEV VDEIRGNHLT BbSF FATI-----E MRRRHAAELD ERIEGWTATQ YGDWLMEALI KAGVAAGEVR DAREAIEDEH LRRRGFWAYL DHPEVGVTLY Cons a $\quad$ i ie a ev e $\quad$ ipc $v$ e dp $r$

POdA PGIVPKLGST PGGVSRPAPT LGQHTDEVLE -QLGIDAGQR AEWRARGLI-

Pfam PGIVPKLGTT PGSVTRSAPT LGQHTAEILG -DLGIDALQQ ADWRQRGII-

BaiF LGLINKFKRN PSEIWRGAPL FGMDNRDILK -DLGYDDAKI DELYEOGIVN EFDLDTTIKR YRLDEVIPHM RKKEE

CaiB PNIMPKFKNN PGQIWRGMPS HGMDTAAILK -NIGYSENDI QELVSKGLAK VED------- - - - - - - - -

FrC VGAPFKFSGF QPEITR-APL LGEHTDEVLK -ELGLDDAKI KELHAKQVV- - -

BbSF NRAPIVESRT PVEMKSAAPS IGQHTREVLG GMLGYSHGEI EDLAAQQVLV -..-.-.-. Cons kf $p$ ei $r$ aP G ht vLk lGyd qi el

Figure 4. Sequence alignment of formyl-CoA transferases. Pfam02515, CoA transferase (WP003209938); Baif, putative cholate CoA transferase (AAC45415); CaiB, $(R)$-carnitine CoA transferase (CAA52112); Frc, formylCoA transferase (AAC45298); and BbsF, $(R)$-benylsuccinate CoA transferase (AAF89841). Identical residues are shown in red; residues suggested to be involved in catalysis are shown in blue. CoA-contacting residues proposed in Frc are marked green.

In this study, we isolated ODB from the surfaces of spinach and strawberries and evaluated their disease control activity. We also explored potential correlations between oxalate-degrading ability and disease inhibitory effects of the ODB isolates. Previous studies have used antagonistic ODB ${ }^{13}$ or ODB with unknown antagonistic effects $^{12}$. Therefore, unlike these previous studies, we attempted to exclude antagonistic effects by excluding $P$. fluorescens ODB5, which exhibited antifungal activity; we selected bacterial strains $M$. zatmanii ODB35 and $P$. abietaniphila ODB36 for further testing of dose response to disease suppression. Significant suppression of $P$. abietaniphila ODB36 against grey mould is shown in Fig. 2. We expect that biological control of fungal pathogens can be achieved by neutralising pathogenic factors and inhibiting the occurrence of resistant individuals by applying a method that does not kill pathogens.

The disease suppression effect of ODB35, which has the highest oxalate-degrading ability, did not reach that of ODB36. This result is likely due to the slow growth rate of ODB35. As shown in Fig. 1, oxalate-degradation activity was calculated by multiplying the $\mathrm{A}_{450}$ value by bacterial growth. ODB35 exhibited relatively slow growth, resulting in a high value. These results suggest that the growth rate of ODB on the plant surface is important to its efficacy as a disease control agent. The aim of this study was to evaluate the oxalate-degrading ability of bacteria in 


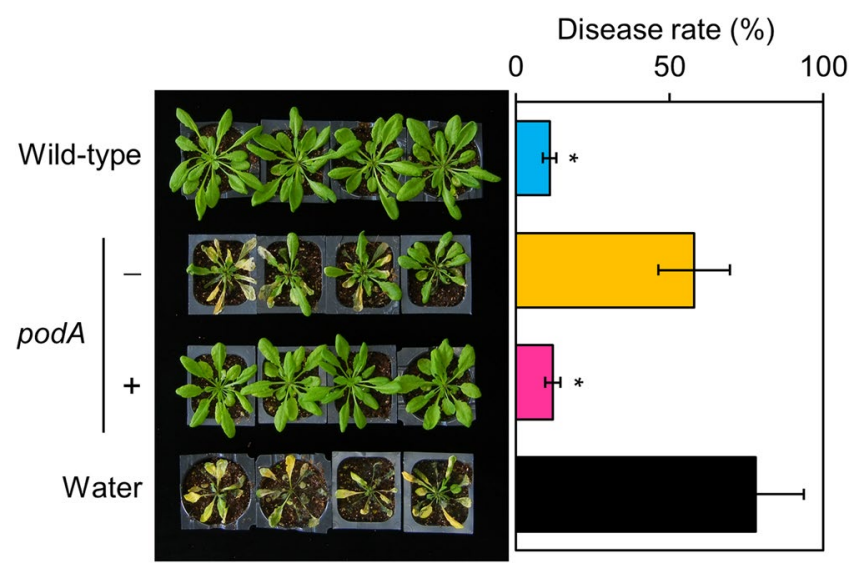

Figure 5. PodA is required for the inhibition of Botrytis infection. Four-week-old Arabidopsis plants were sprayed with suspensions $\left(\mathrm{OD}_{600}\right.$ value 1.0$)$ of the wild-type ODB36, podA- mutant $(-)$, and podA complementation $(+)$ strains. After incubation for 2 days, a $B$. cinerea spore suspension $\left(5 \times 10^{5} \mathrm{spores} / \mathrm{mL}\right)$ was prepared using sterilised water. The spore suspension was sprayed until run-off occurred. Inoculated plants were placed in an opaque plastic box lined with saturated paper towels, the lids were removed after 2 days, and disease development was observed for 12 days and measured. Wild-type ODB36 strain exhibited significant disease suppression. The $p o d A^{-}$mutant did not inhibit Botrytis infection; the defect was recovered by podA complementation. Values are averages of duplicate assays; error bars represent the range. Asterisks denote significant differences from the water control (*p $<0.05$; Student's $t$ test).

a

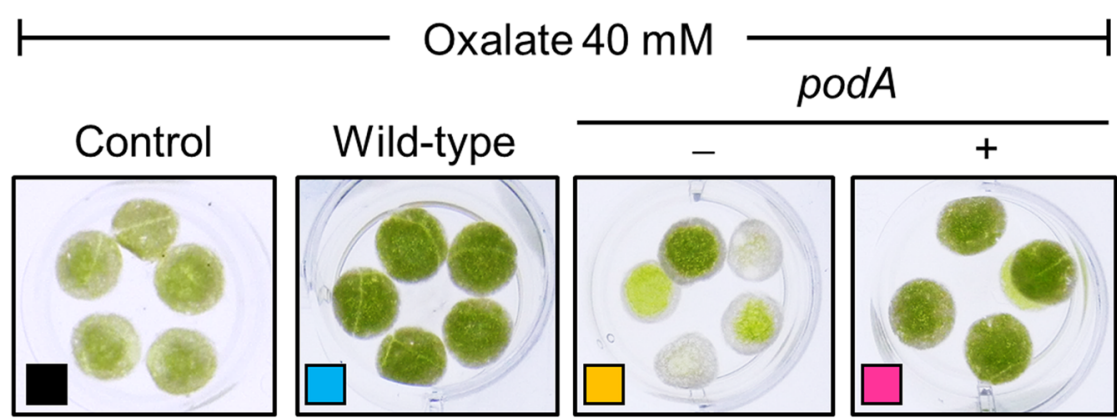

b

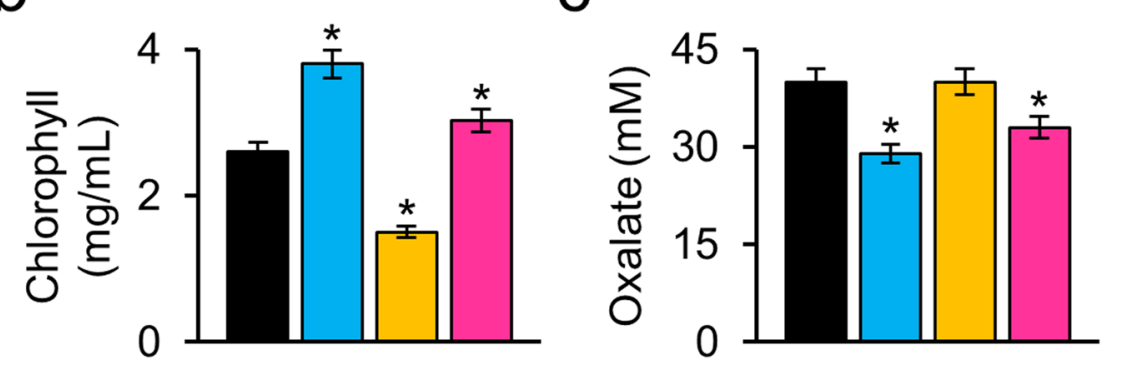

Figure 6. PodA is required for the inhibition of oxalate toxicity. (a) Tobacco leaf disc assays. Leaf discs were placed in suspensions of the wild-type, $p o d A^{-}$mutant $(-)$, and podA complementation $(+)$strains supplemented with $40 \mathrm{mM}$ sodium oxalate. After incubation for 5 days, oxalate toxicity was observed on the leaf discs in the pod $A^{-}$mutant suspension but was reversed by podA complementation. (b) Chlorophyll in the leaf discs (a) was measured as described in the Methods section. (c) Remaining oxalate in suspensions (a) of the wild-type ODB36, pod $A^{-}$mutant, and podA complementation strains. Values are averages of duplicate assays; error bars represent the range. Asterisks denote significant differences from the control $\left({ }^{*} p<0.05\right.$; Student's $t$ test). 
a

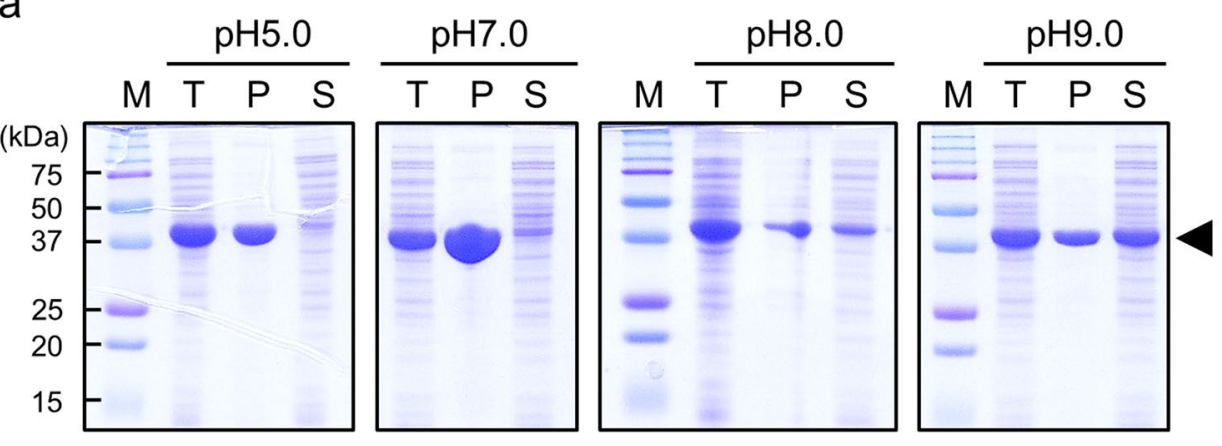

b

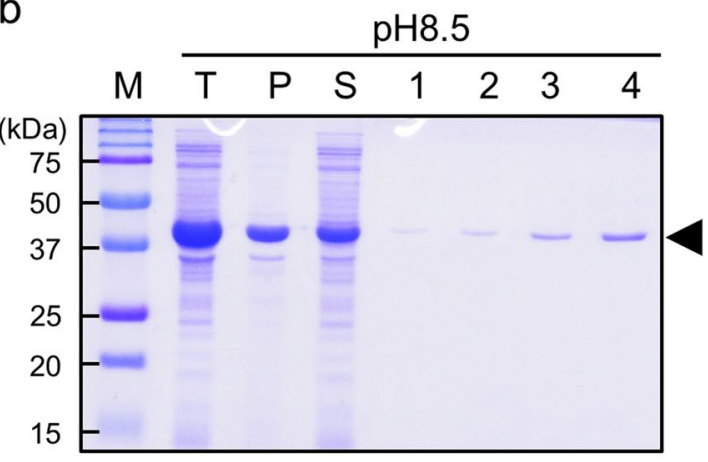

C

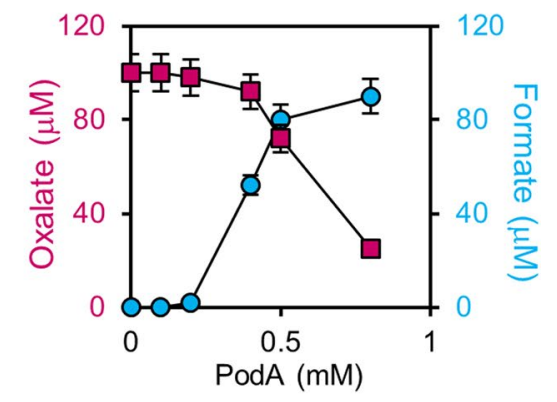

Figure 7. Overexpression, purification, and oxalate-degrading and formate conversion activities of PodA-His. (a) Sodium dodecyl sulphate polyacrylamide gel electrophoresis (SDS-PAGE) of PodA-His expression. The centrifuged cells of E. coli BL21(DE3) expressing PodA-His protein were subjected to sonic oscillation in cold $200 \mathrm{mM}$ potassium phosphate buffer at $\mathrm{pH}$ 5.0-9.0. Each $10 \mu \mathrm{L}$ sample was dissolved in $10 \mu \mathrm{L}$ of $2 \times$ loading buffer and boiled for $3 \mathrm{~min}$ at $100^{\circ} \mathrm{C}$. Final volumes of $10 \mu \mathrm{L}$ were loaded in each lane. $\mathrm{M}$, molecular mass standards; T, total cell extracts; P, pellet; S, supernatant of BL21(DE3) harbouring pLY205 after IPTG induction. (b) SDS-PAGE of PodA-His purification. Cell free sonic extract was worked up for purification using a Ni-NTA spin column. The purified protein eluted with $0.5 \mathrm{M}$ imidazole was dialysed. Lanes $1-4: 1-, 2-, 5-$, and $10-\mu \mathrm{L}$ final elutions, respectively, containing PodA-His. Separation was conducted by $10 \%$ SDS-PAGE. Bands were visualised after staining with Coomassie Blue. The band corresponding to PodA-His is indicated. (c) Oxalatedegrading (closed square) and formate conversion (closed circle) activities of PodA-His determined using an oxalate colorimetric assay kit and a formate colorimetric assay kit, respectively. Values are averages of duplicate assays; error bars represent the range.

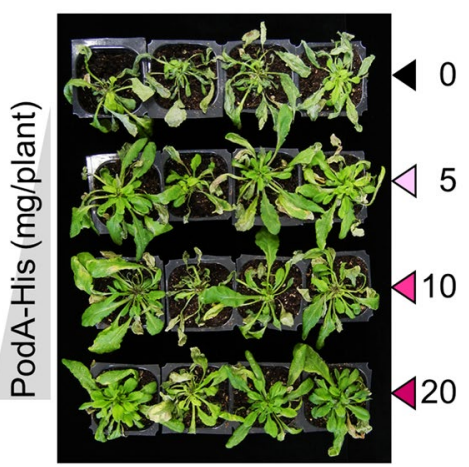

\section{Oxalate toxicity (\%)}

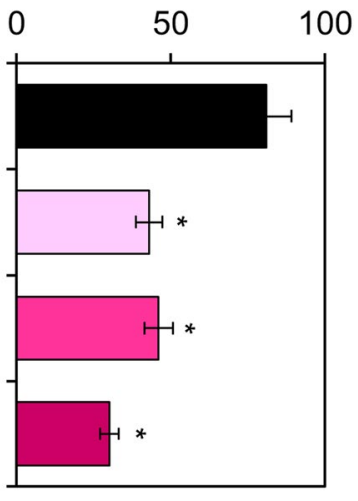

Figure 8. Inhibition of oxalate toxicity by PodA-His. Image captured 5 days after inoculation; oxalate toxicity rate (\%). Six-week-old Arabidopsis plants were sprayed with PodA-His suspension. After incubation for 1 day, the plants were sprayed with an oxalate suspension $(20 \mathrm{mM} ; 1 \mathrm{~mL} /$ plant $)$. Oxalate toxicity was monitored for 5 days. PodA-His exhibited considerable and concentration-dependent inhibition of oxalate toxicity. Values are averages of duplicate assays; error bars represent the range. Asterisks denote significant differences from the control $(* p<0.05$; Student's $t$ test). 


\section{Oxalate}

$+$

Formyl-CoA

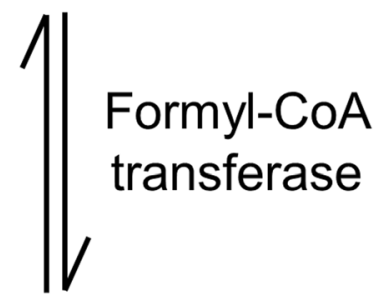

\section{Oxalyl-CoA \\ $+$ \\ Formate}

Figure 9. Oxalate degradation by formyl-CoA transferase. The two substrates, oxalate and formyl-CoA, are converted into the two products formate and oxalyl-CoA.

disease control; we finally selected ODB36 as the optimal agent among those examined. Growth on plant surfaces was not investigated in this study.

Interestingly, the wild-type and podA complementation strains eliminated the toxicity of $40 \mathrm{mM}$ oxalate, such as bleaching of tobacco leaf discs, but the pod $A^{-}$mutant showed bleaching due to no oxalate toxicity. The remaining oxalate concentration in the wild-type and $p o d A^{-}$complementation strains was lower than that of the podA mutant strain. These data suggest that podA contributes to the degradation of oxalate. These results are in good agreement with the finding that grey mould control is by oxalate degradation in P. abietaniphila.

Family III CoA transferases include formyl-CoA:oxalate CoA transferase ${ }^{16}$, succinyl-CoA: $(R)$-benzylsuccinate CoA transferase ${ }^{19},(E)$-cinnamoyl-CoA: $(R)$-phenyllactate CoA transferase ${ }^{20}$, and butyrobetainyl-CoA: $(R)$-carnitine $\mathrm{CoA}$ transferase ${ }^{21}$. These CoA transferases occur in prokaryotes and eukaryotes and catalyze CoA transfer reactions in a highly substrate- and stereo-specific manner ${ }^{22}$. Additionally, the PodA exhibited $81 \%$ identity $(89 \%$ similarity) with Pfam02515, and 30\% identity (47\% similarity) with O. formigenes formyl-CoA transferase, which was the first member of family III of CoA transferases to be characterized ${ }^{22}$.

In this study, we did not quantify the formate concentration in ODB because P. abietaniphila strains contain several genes encoding formate dehydrogenase subunits, which catalyse the oxidation of formate to carbon dioxide. However, we performed protein overexpression and enzyme activity assays and confirmed that PodA-His converted oxalate to formate, which plays a role in the biosynthesis of many compounds in energetic metabolism and signal production related to stress in plants ${ }^{23}$.

In plants, three types of oxalate-degrading enzymes are known, namely, oxidase (OXO), decarboxylase (OXDC), and acetylases, among which four enzymes (oxalyl-CoA synthetase [AAE3], oxalyl-CoA decarboxylase [OXDE], formyl-CoA hydrolase [FXH], and formyl-CoA dehydrogenase [FXDE]) act in stages ${ }^{24-26}$. In enzymatic analysis of oxalate degradation of bacteria, formyl-CoA transferase (EC 2.8.3.16) catalyzes the chemical reaction, which is demonstrated in Fig. $9^{15,16}$. The two substrates, oxalate and formyl-CoA, are converted into the two products oxalyl-CoA and formate. Formyl-CoA is required for the conversion of oxalate to oxalyl-CoA by formyl-CoA transferase ${ }^{16}$. However, the inhibitory effect of PodA on oxalate toxicity in Arabidopsis suggests the presence of formyl-CoA as a substrate. It is possibile that formyl-CoA derived from Arabidopsis-resident microorganisms acts as a substrate for PodA.

Previous studies have explored disease control in transgenic crops using plant-derived oxalate-degrading genetic resources. Barley oxalate oxidase transgenic peanuts showed enhanced resistance against Sclerotinia minor $^{27}$. Soybean plants expressing wheat oxalate oxidase were resistant to Sclerotinia sclerotiorum ${ }^{28,29}$. These preliminary studies suggest that developed oxalate degradative genetic resources can be introduced into crops and inspire the development of transgenic plants with related abilities.

In conclusion, to overcome the competing processes of biological control and occurrence of resistant species, we investigated non-antimicrobial genetic resources for biological control of plant pathogens. Oxalate, a key pathogenic factor of necrotrophic fungi, was targeted to disarm, but not kill, plant pathogens. Oxalate-degrading bacteria were isolated and identified, and their activity was evaluated. Using disease suppression assays, 
P. abietaniphila ODB36 was finally selected for further investigation. PodA from ODB36, which encodes formyl-CoA transferase, was overexpressed and assayed using oxalate degradation tests. Bacterial cells of both ODB36 and enzyme PodA were found to be directly applicable in the field to control grey mould. The podA gene can also be applied for the development of transgenic resistant cultivars against grey mould.

\section{Methods}

Isolation of ODB. Surfaces of strawberries and spinach were swabbed with sterilised swabs, which were then streaked onto OM medium with $1.5 \%$ agar and incubated at $28^{\circ} \mathrm{C}$. After 7 days, single colonies were re-streaked onto OM agar medium for purification.

Bacterial oxalate-degrading activity. Oxalate-degrading activity was evaluated using an oxalate colorimetric assay kit according to the supplier's protocol. Optical density $\left(\mathrm{OD}_{600}\right)$ was measured in bacterial cultures grown in $\mathrm{OM}$ broth plus $1 / 10 \mathrm{TSB}$ at $28^{\circ} \mathrm{C}$ for 3 days. After centrifugation, the supernatants were transferred into 96-well plates and mixed with $2 \mu \mathrm{L}$ oxalate converter, which was supplied with the kit. The mixtures were incubated at $37^{\circ} \mathrm{C}$ for $1 \mathrm{~h}$, and $50 \mu \mathrm{L}$ prepared reaction mix ( $46 \mu \mathrm{L}$ buffer; $2 \mu \mathrm{L}$ oxalate enzyme mix, and $2 \mu \mathrm{L}$ oxalate probe; all reagents were supplied with the kit) was added to each well containing standard or samples. Then the plates were incubated at $37^{\circ} \mathrm{C}$ for $1 \mathrm{~h}$ and absorbance was measured at $450 \mathrm{~nm}\left(\mathrm{~A}_{450}\right)$. The values reporting the amount of oxalate remaining in the sample were normalized to culture growth by multiplying the $\mathrm{A}_{450}$ value for the non-degraded oxalate amount by the $\mathrm{OD}_{600}$ of the culture. The lower the value, the higher the degradation activity.

16S rRNA gene sequencing. To confirm the identities of the ODB, the 16S rRNA gene was amplified and sequenced using the primers $27 \mathrm{mF}$ (5'-AGAGTTTGATCMTGGCTCAG-3 $3^{\prime}$ ) and $1429 \mathrm{mR}$ (5'-GGYTACCTTGTTACGACTT-3'). Total DNA was extracted using the Wizard Genomic DNA Purification Kit (Promega, Madison, WI, USA) following the manufacturer's instructions. Polymerase chain reaction (PCR) was performed using a T100 thermal cycler (Bio-Rad, Hercules, CA, USA) with PCR polymerase (AccurPower PCR Premix; Bioneer, Daejeon, South Korea), $1 \mu \mathrm{L}$ target DNA, and $1 \mathrm{mM}$ each primer at $98^{\circ} \mathrm{C}$ for $2 \mathrm{~min}$, followed by 30 cycles of denaturation at $98^{\circ} \mathrm{C}$ for $30 \mathrm{~s}$, annealing at $55^{\circ} \mathrm{C}$ for $30 \mathrm{~s}$, and extension at $70^{\circ} \mathrm{C}$ for $1 \mathrm{~min}$, followed by a final extension at $72^{\circ} \mathrm{C}$ for $4 \mathrm{~min}$. The amplified products were separated by electrophoresis in $0.8 \%$ (w/v) agarose gels. PCR amplification yielded a single visible DNA product, whose band was cleaved from the ethidium bromide (EtBr)-stained gel and purified using a 200-p Expin Gel SV kit (GeneAll biotechnology, Seoul, Korea), following the manufacturer's instructions. Purified PCR products were sequenced by Macrogen Services (Daejeon, Korea) in both directions using previously described primers ${ }^{30}$. DNA sequences were analyzed using the BLASTn program. DNA sequences of the 16S rRNA gene were compared with those in the National Center for Biotechnology Information (NCBI) GenBank database (http://www.ncbi.nlm.nih.gov/blast/).

Antifungal activity test. Among the ODB, the antifungal activity of the three isolates (ODB5, ODB35, and ODB36) showing the highest oxalate-degrading activity was tested against B. cinerea, A. alternata, and S. cerevisiae. Botrytis cinerea and A. alternata spores were spread on half potato dextrose agar with half protease peptone (PDP) and 1.5\% agar. Saccharomyces cerevisiae cells were embedded in PDP agar. The ODB suspension $(10 \mu \mathrm{L})$ was dropped onto the plate, which was then incubated at $28^{\circ} \mathrm{C}$ for $48 \mathrm{~h}$ and the magnitude of inhibition of fungal growth was monitored.

Disease suppression assay. Arabidopsis thaliana Columbia (Col-0) was grown in a growth chamber at $20^{\circ} \mathrm{C}$ under a $16-\mathrm{h}$ photoperiod. Bacterial suspensions were prepared by resuspending the strains using sterilised water to adjust the $\mathrm{OD}_{600}$ values to the corresponding ranges (0.7-1.0). We sprayed 3-week-old Arabidopsis plants with the ODB suspensions ( $5 \mathrm{~mL} /$ plant). After 2 days of incubation, a $B$. cinerea spore suspension $\left(5 \times 10^{5}\right.$ spores/ $\mathrm{mL}$ ) was prepared using sterilised water. The spore suspension was sprayed until run-off. Inoculated plants were placed in an opaque plastic box lined with saturated paper towels, the lids were removed after 2 days, and disease development was observed for 16 days and measured according to the following calculation: (no. diseased leaves/ no. inoculated leaves) $\times 100$.

Sequence alignment and phylogenetic analysis. Protein sequences obtained from NCBI (https:// www.ncbi.nlm.nih.gov/) were aligned and utilized to generate an unrooted phylogenetic tree using the neighbor-joining method (CLUSTALX software).

Construction of the podA $A^{-}$mutant and podA complementation strains. To generate the pod $A^{-}$mutant, plasmid pLY201, carrying the internal fragment of the podA gene was constructed using ODB36 genomic DNA as the PCR template and PodAE (5'-AAGATACTGGGTGAGTTT-3') and PodAK (5'-GGTACCCATCACCAGTTTCGGATT-3') as primers. The amplified region (291 bp) was purified from an agarose gel and ligated into the pGEM-T Easy Vector System (Promega, Mannheim, Germany) to generate pLY201, which was confirmed by sequencing. pLY201 fragments generated by digestion with the restriction enzymes EcoRI and KpnI (TaKaRa Bio Inc., Kusatsu, Japan) were purified after electrophoresis from an agarose gel and inserted into the suicide vector pVIK112 $2^{31}$, generating pLY207. The resulting construct, pLY207, was transferred into E. coli S17-1 $\lambda$ pir and introduced into $P$. abietaniphila ODB36 by conjugation, generating podAlac ZY. The lacZY reporter gene fusion insertion mutants were selected based on the kanamycin-resistance phenotype and confirmed by PCR with primers that annealed upstream of the truncated fragments of podA, PodPro (5'-ATGCAAAAGCCTTTGCAAGGG-3') and LacFuse (5'-GGGGATGTGCTGCAAGGCG-3').

To construct the podA complementation strain, wild-type coding sequences with Plac were cloned into the broad-host vector pLAFR3. Coding sequences were amplified by PCR from ODB36 genomic DNA, using the 
primers PodAH (5'-GGCAAGCTTCTGAAACAGGAAACAGCTATGCAAAAGCCTTTGCAAGGG-3') and PodAP (5'-GGCCTGCAGTCAAATCAATCCGCGAGCGCG-3') and Phusion polymerase. Amplicons were ligated into pGEM-T Easy, confirmed by sequencing, excised by restriction enzyme cleavage, and ligated with appropriately cleaved pLAFR3. Plasmid pLY233 harbouring Plac-podA was verified by restriction digestion and sequencing prior to conjugation with $P$. abietaniphilae cells.

Leaf disc assays and chlorophyll quantification. Tobacco leaf disc assays ${ }^{32}$ and chlorophyll quantification assays ${ }^{33}$ were performed as described previously.

Overexpression and purification of PodA-His protein. We overexpressed PodA in E. coli by amplifying the coding region of podA using chromosomal DNA of ODB36 as a template with the primers PodN (5'-GGCCATATG CAAAAGCCTTTGCAAGGG-3') with initiating ATG (bold) and PodX (5'-GGCCTCGAGAATC AATCCGCGAGCGCGCCA-3') omitting stop codon, to which NdeI and XhoI sites (underline) were added. The amplified product was cloned at NdeI/XhoI site of pET21b containing a six-His tag at C-terminal (Novagen, Darmstadt, Germany) to generate pLY205. PodA-His was overexpressed in E. coli BL21(DE3), as described by the manufacturer (Novagen, Darmstadt, Germany). Cell free extracts were prepared by subjecting a suspension of $5 \mathrm{~g}$ centrifuged cells (wet weight) in $40 \mathrm{~mL}$ cold $0.2 \mathrm{M}$ potassium phosphate or $0.2 \mathrm{M}$ glycine- $\mathrm{NaOH}$ buffer ( $\mathrm{pH}$ 5.0-9.0) to sonic oscillation in a solicitor (VCX130; Sonics \& Materials Inc., Newtown, CT, USA) for 5 min at $4^{\circ} \mathrm{C}$. The sonic extract was centrifuged at $4{ }^{\circ} \mathrm{C}$ and $10,000 \times g$ for $10 \mathrm{~min}$. The supernatant solution was worked up for purification using a Ni-NTA spin column (Qiagen, Valencia, CA, USA). The eluted protein was dialyzed with the same buffer used for sonic oscillation, and the concentration of the purified protein was measured via the Bradford method with bovine serum albumin as the standard ${ }^{34}$.

Oxalate degradation and formate conversion activities of PodA-His. The oxalate-degrading and formate conversion activities of purified PodA-His were evaluated using an oxalate colorimetric assay kit and a formate colorimetric assay kit, respectively, according to the supplier's protocol (BioVision Inc., Milpitas, CA, USA).

Inhibition of oxalate toxicity by PodA-His protein. Arabidopsis thaliana Columbia (Col-0) was grown in a growth chamber at $20^{\circ} \mathrm{C}$ under a 16 -h photoperiod. Protein suspensions were prepared by resuspending PodA-His in potassium phosphate buffer ( $\mathrm{pH} 8.5)$ to 1,2 , and $4 \mathrm{mg} / \mathrm{mL}$. We sprayed 6-week-old Arabidopsis plants with PodA-His protein suspensions ( $5 \mathrm{~mL} /$ plant). After incubation forl day, the plants were sprayed with a sodium oxalate suspension $(20 \mathrm{mM} ; 1-\mathrm{mL} /$ plant). Oxalate toxicity was observed for 5 days and calculated according to the following equation: (no. of bleached leaves/total no. of leaves) $\times 100$.

Received: 16 September 2019; Accepted: 17 January 2020;

Published online: 31 January 2020

\section{References}

1. Wiliamson, B., Tudzynski, B., Tudzynski, P. \& van Kan, J. A. Botrytis cinerea: the cause of grey mould disease. Mol. Plant. Pathol. 8, $561-580$ (2007).

2. Maude, R. B. Disease control. In The biology of Botrytis. (eds. Coley-Smith, J. R., Verhoeff, K. \& Jarvis, W. R.). 275-308 (London: Academic Press, 1980).

3. Burgess, D. R., Bretag, T. W. \& Keane, P. J. Seed to seedling transmission of Botrytis cinerea in chickpea ad disinfestation of seed with moist heat. Aust. J. Exp. Agric. 37, 223-229 (1997).

4. Komárek, M., Čadková, E., Chrastný, V., Bordas, F. \& Bollinger, J. C. Contamination of vineyard soils with fungicides: a review of environmental and toxicological aspects. Env. Inter. 36, 138-151 (2010).

5. Leroux, P. et al. Mechanisms of resistance to fungicides in field of Botrytis cinerea. Pest. Manag. Sci. 58, 876-888 (2002).

6. Haidar, R., Fermaud, M., Calvo-Garrido, C., Roudet, J. \& Deschamps, A. Modes of action for biological control of Botrytis cinerea by antagonistic bacteria. Phytopathol. Mediterr. 55, 301-322 (2016).

7. Gabler, F. M. \& Smilanick, J. L. Postharvest control of table grape gray mold on detached berries with carbonate and bicarbonate salts and disinfectants. Am. J. Enol. Vitic. 52, 12-20 (2001).

8. Rollins, J. A. \& Dickman, M. B. pH signaling in Sclerotinia sclerotiorum: Identification of a pacC/RIMI homolog. Appl. Env. Microbiol. 67, 75-81 (2001).

9. Murru, N. et al. Screening of oxalate degrading lactic acid bacteria of food origin. Ital. J. Food Saf. 6, 61-64 (2017).

10. Federici, F. et al. Characerization and heterologous expression of the oxalyl coenzyme A decarboxylase gene from Bifidobacterium lactis. Appl. Env. Microbiol. 70, 5066-5073 (2004).

11. Manteau, S., Abouna, S., Lambert, B. \& Legendre, L. Differential regulation by ambient $\mathrm{pH}$ of putative virulence factor secretion by the phytopathogenic fungus Botrytis cinerea. FEMS Microbiol. Ecol. 43, 359-366 (2003).

12. Nagarajkumar, M., Jayaraj, J., Muthukrishnana, S., Bhaskaran, R. \& Velazhahan, R. Detoxification of oxalic acid by Pseudomonas fluorescens strain PfMDU2: Implications for the biological control of rice sheath blight caused by Rhizoctonia solani. Microbiol. Res. 160, 291-298 (2005).

13. Schoonbeek, H. J., Jacquat-Bovet, A. C., Mascher, F. \& Métraux, J. P. Oxalate-degrading bacteria can protect Arabidopsis thaliana and crop plants against Botrytis cinerea. Mol. Plant-Microbe Interact. 20, 1535-1544 (2007).

14. Hatch, M. \& Freel, R. W. Alterations in intestinal transport of oxalate in disease states. Scanning Microsc. 9, 1121-1126 (1995)

15. Azcarate-Peril, M. A., Bruno-Bárcena, J. M., Hassan, H. M. \& Klaenhammer, T. R. Transcriptional and functional analysis of oxalylCoenzyme A (CoA) decarboxylase and formyl-CoA transferase genes from Lactobacillus acidophilus. Appl. Env. Microbiol. 72, 1891-1899 (2006).

16. Jonsson, S., Ricagno, S., Lindqvist, Y. \& Richards, N. G. Kinetic and mechanistic characterization of the formyl-CoA transferase from Oxalobacter formigenes. J. Biol. Chem. 279, 36003-36012 (2004)

17. Martin, V. J. \& Mohn, W. W. Genetic investigation of the catabolic pathway for degradation of abietane diterpenoids by Pseudomonas abietaniphila BKME-9. J. Bacteriol. 182, 3784-3793 (2000).

18. Kim, W.-I. et al. Inhibition of Salmonella enterica growth by competitive exclusion during early alfalfa sprout development using a seed-dwelling Erwinia persicina strain EUS78. Intl J. Food Microbiol. 312, 108374 (2020). 
19. Leutwein, C. \& Heider, J. Succinyl-CoA:(R)-benzylsuccinate CoA-transferase: an enzyme of the anaerobic toluene catabolic pathway in denitrifying bacteria. J. Bacteriol. 183, 4288-4295 (2001).

20. Dickert, S., Pierik, A. J., Linder, D. \& Buckel, W. The involvement of coenzyme A esters in the dehydration of (R)-phenyllactate to (E)-cinnamate by Clostridium sporogenes. Euro J. Biochem. 267, 3874-3884 (2000).

21. Rangarajan, E. S., Li, Y., Iannuzzi, P., Cygler, M. \& Matte, A. Crystal structure of Escherichia coli crotonobetainyl-CoA: carnitine CoA-transferase (CaiB) and its complexes with CoA and carnitinyl-CoA. Biochem. 44, 5728-5738 (2005).

22. Heider, J. A new family of CoA-transferases. FEBS Lett. 509, 345-349 (2001).

23. Igamberdiex, A. U., Bykova, N. V. \& Kleczkowski, L. A. Origins and metabolism of formate in higher plants. Plant. Physiol. Biochem. 37, 503-513 (1999).

24. Anantharam, V., Allison., M. J. \& Maloney, P. C. Oxalate: formate exchange. basis energy coupling Oxalobacter. J. Bio Chem. 264, 7244-7250 (1989).

25. Turroni, S. et al. Oxalate consumption by lactobacilli: evaluation of oxalyl-CoA decarboxylase and formyl-CoA transferase activity in Lactobacillus acidophilus. J. Appl. Microbiol. 103, 1600-1609 (2007).

26. Zhao, C. et al. Oxalate-degrading enzyme recombined lactic acid bacteria strains reduce hyperoxaluria. Urol. 113, 253.e1-253.e7 (2018).

27. Livingstone, D. M., Hampton, J. L., Phipps, P. M. \& Grabau, E. A. Enhancing resistance to Sclerotinia minor in peanut by expressing a barley oxalate oxidase gene. Plant. Physiol. 137, 1354-1362 (2005).

28. Donaldson, P. A., Anderson, T., Lane, B. G., Davidson, A. L. \& Simmonds, D. H. Soybean plants expressing an active oligomeric oxalate oxidase from the wheat gf- 2.8 (germin) gene are resistant to the oxalate-secreting pathogen Sclerotinia sclerotiorum. Physiol. Mol. Plant. Pathol. 59, 297-230 (2001).

29. Yang, X. et al. Enhanced resistance to sclerotinia stem rot in transgenic soybean that overexpresses a wheat oxalate oxidase. Transgenic Res. 28, 103-114 (2019).

30. Weisburg, W. G., Barns, S. M., Pelletier, D. A. \& Lane, D. J. 16S ribosomal DNA amplification for phylogenetic study. J. Bacterial 179, 697-703 (1991)

31. Kalogeraki, V. S. \& Winans, S. C. Suicide plasmids containing promoterless reporter genes can simultaneously disrupt and create fusions to target genes of diverse bacteria. Gene 188, 69-75 (1997).

32. Koh, S. et al. A novel light-dependent selection marker system in plants. Plant. Biotechnol. J. 9, 348-358 (2011).

33. Amon, D. I. Copper enzymes in isolated chloroplasts. Polyphenoloxidase in Beta vulgaris. Plant. physiol. 24, 1-15 (1949).

34. Bradford, M. M. A rapid and sensitive method for the quantitation of microgram quantities of protein utilizing the principle of protein-dye binding. Anal. Biochem. 72, 248-254 (1976).

\section{Acknowledgements}

This work was supported by the Gyeongsang National University Fund for Professors on Sabbatical Leave, 2017. Additional funds were provided by Basic Science Research Program through the National Research Foundation of Korea (NRF) funded by the Ministry of Education (2015R1A6A1A03031413).

\section{Author contributions}

J.K. conceived and designed research. Y.L. and O.C. conducted experiments. B.K., J.B. and S.K. analyzed data. Y.L. and J.K. wrote the manuscript. All authors read and approved the manuscript.

\section{Competing interests}

The authors declare no competing interests.

\section{Additional information}

Supplementary information is available for this paper at https://doi.org/10.1038/s41598-020-58609-z.

Correspondence and requests for materials should be addressed to J.K.

Reprints and permissions information is available at www.nature.com/reprints.

Publisher's note Springer Nature remains neutral with regard to jurisdictional claims in published maps and institutional affiliations.

(c) (i) Open Access This article is licensed under a Creative Commons Attribution 4.0 International

License, which permits use, sharing, adaptation, distribution and reproduction in any medium or format, as long as you give appropriate credit to the original author(s) and the source, provide a link to the Creative Commons license, and indicate if changes were made. The images or other third party material in this article are included in the article's Creative Commons license, unless indicated otherwise in a credit line to the material. If material is not included in the article's Creative Commons license and your intended use is not permitted by statutory regulation or exceeds the permitted use, you will need to obtain permission directly from the copyright holder. To view a copy of this license, visit http://creativecommons.org/licenses/by/4.0/.

(C) The Author(s) 2020 